PART V

GENERAL CONCLUSION AND FINAL DISCUSSION 


\title{
DO STELLAR SYSTEMS AGREE WITH THE SINGULAR EVOLUTION OF $N$-BODY SYSTEMS?
}

\author{
D. LYNDEN-BELL \\ Institute of Astronomy, The Observatories, Cambridge, U.K.
}

One of the important jobs of scientists is to formulate new laws and principles, and I have attempted to summarise those we have learnt in this most enjoyable and fruitful symposium in the laws stated below. However, recognising that laws derived from stellar dynamics may lead to important discoveries in other fields, I have translated these laws into the language of sociology. The key concept of hard and soft binaries has been introduced into stellar dynamics by Heggie. If $\left\langle\frac{1}{2} m v^{2}\right\rangle$ at some point in a stellar system is defined to be $\frac{3}{2} \beta^{-1}$ then Heggie defines a soft binary to be one whose internal energy has $\beta|\varepsilon|<1$ and a hard binary to be one with $\beta|\varepsilon|>1$. Heggie's laws cover the interaction of these binaries with the field stars and his first basic discovery is that on interacting with this society hard binaries become harder while soft binaries become softer and may even cease to be binaries at all. The same may be true of criminals in their interaction with society.

Heggie's first law. The rate of hardening of a criminal is independent of his hardness once he has become a hard criminal.

Heggie's second law. Very hard criminals are locked up (by adiabatic invariants) most of the time so they only harden by committing relatively few but very violent crimes, while less hard criminals commit minor crimes more often.

Aarseth's law. Small societies evolve until they are dominated by a central very hard criminal who may expel other members of society, but he may eventually be displaced from his central position or even ejected altogether after a violent interaction with another member of society.

Hénon's law. Every society eventually evolves a central core or clique that dominates it, but when the clique gets too small it may become dominated by a hard criminal just as Aarseth's law describes.

Spitzer's law. Heavyweight individuals jostle their way through society until they join the ruling clique which is the ultimate source of power. Unfortunately power corrupts, and they are also the most likely to become hard criminals.

Hénon's conjecture is that a certain amount of crime is needed by society and if insufficient crime is committed then the ruling clique will shrink until a fresh hard criminal is created by the more prickly interactions among the smaller agitated clique.

The agreement between the rates of evolution calculated by $N$ body computations and by the Monte Carlo approaches of Hénon and Spitzer is impressive as Wielen showed, and the refinement of this agreement by Hénon should convince us that the Monte Carlo method is accurate, at least until the number of stars in the central core 
becomes so few that two body encounters leading to escape become frequent. When the number of stars in the core is only 100 or so the $N$ body integrations become more powerful in giving us a detailed description of what happens. An area that needs far more study is the correct boundary condition to apply at the junction between this small core and the rest of the cluster. Is Hénon's conjecture - that the rest of the cluster needs a certain energy supply and that a central binary in the core will be formed and supply energy at just this rate - correct? If so, how should this energy demand by the rest of the cluster be represented when the $N$ body calculation of the core is performed? Perhaps the boundary conditions adopted may be open to check by the powerful new $N$ body method of Ahmad and Cohen.

If we take King's advice and turn to the great book of Nature for final confirmation that these cores have developed and on the expected timescale, we find our difficulties are not over. In globular clusters where we expect that the cores must have formed quite long ago, he sees no sign of a central nucleus, while in galaxies where there is no time for the stellar dynamical processes to act we see the sort of nuclei that we might have expected for the globulars. Of course, we may be able to wriggle out of this - the predicted cores should not contain a large proportion of the mass or light, only a significant fraction of the binding energy, so perhaps the core radius as defined by King has little to do with the cores as predicted by Monte Carlo calculations which have shrunk to be of negligible mass. My present belief is that some sort of funnel going up to a core of high density with one or more binary stars at its centre ought to be seen. However, we must bear in mind that globulars must have had massive stars in them at one time and their whole history may have been quite complicated. Let us turn back to the theories and simulations to see what we expect to happen once the core has developed to very great densities etc. There is little doubt that the contraction of the core leads to the creation of Aarseth's central hard binary. However, for a globular cluster as it is to-day a single pair probably cannot hold an energy of $\frac{1}{10}$ of the cluster energy, even with the two stars almost touching. Furthermore, such a binary will wander, due to the recoil whenever it gives up energy to a third star. The critical question is whether this recoil is sufficient to send it out of the cluster, as often happens in the numerical simulations of 100 stars when the stars have equal mass. As it hardens due to such encounters one might expect its escape but for the fact that the surrounding core will be getting deeper and deeper, and so the internal energy of the binary may only keep pace with the increasing temperature of the few stars left in the core. Thus the central binary may merely wander with translational energy $\frac{3}{2} k T$ like the remaining stars. In any case the central binary will occasionally eject a star through a violent encounter so there will be mass loss from the centre. In clusters of unequal mass the central hard binary will be heavy, and this will help to keep it fixed at the centre, and it will eject those lighter stars that have close encounters. However, at this point stellar evolution probably intervenes for the heavy stars will blow up; their debris will leave the cluster and the way will be open for another heavy binary to form. It is an interesting question to know whether the core evolution has to start all over again once its dominating binary leaves, or whether a sufficient funnel leading to the core has 
remained so that a new binary can form almost at once. The only heavy bodies that we expect to be stable for long times will be the black holes. If massive ones are made we must expect dynamical friction to bring them back to the central regions, provided that they have remained bound to the cluster.

Turning now to galaxies and clusters of galaxies, we have our first difference in that the galaxies are relatively large and fragile, so that their close encounters are dissipative. Work by Alladin, and more recently by Toomre, shows clearly the possibility of coalescence and the nuclei will wind their way down to the centre by dynamical friction. A number of people have speculated that the stars thrown off in these processes may be the origin of the envelopes of the $\mathrm{cD}$ galaxies. One wonders whether the two nuclei will eventually form a strong central binary and if so what will happen as more nuclei collect at the centre. If we were to apply our $N$ body experience to a giant elliptical ignoring the wrong time-scale, it is interesting to note that a core should develop with a binding energy $\frac{1}{10}$ of the whole. This core energy will be concentrated into few degrees of freedom and must be $10^{60} \mathrm{erg}$ for a large galaxy, sufficient for a large radio outburst. It was a bit sad to see that the 3 massive body model of Valtonen and Saslaw was in difficulty with the rarity of the correct initial conditions.

I turn now to haloes. I was impressed by Freeman's negative result on NGC 253 and his emphasis on the heavy lens components of some SO galaxies. Could it be that we have central disc components with large $Q=\left\langle V_{R}^{2}\right\rangle^{1 / 2} K /(3.36 G \mu)$. Here $\mu(R)$ is the surface density of the galaxy and $K(R)$ is the epicyclic frequency. I note that Hohl's thorough discussion of bar instabilities in his numerical experiments did not include experiments with large $Q$ in the middle, and that large $Q$ there would be most useful in satisfying Ostriker and Peeble's criterion. Furthermore, that is just the place where every astronomer expects high random motion. The $\mathbf{M}$ dwarf problems discussed by Schmidt and Biermann seems to be more and more intriguing. We would probably patch things up if high central $Q$ would allow us a $Q$ of just over 1 out here, but even then we have the basic problem of the very flat layer of stars that are probably old. King's report on velocities of five stars and their lack of $\mathrm{H}$ and $\mathrm{K}$ emission certainly adds weight to the case already made by Sanduleak, Murray and Weistrop. It seems to me that we need haloes very badly in the system with large central bulges and the ellipticals and that large haloes may not be necessary to stabilise the flat Sc's. In the clusters of galaxies large haloes accompanying all galaxies with strong central bulges are probably all that is necessary.

We will hear more of the importance of viscosity in the formation of the galaxy as introduced by Larson, and all of us will remember Brahic's fascinating talk on the role of collisions and dissipation in all of astrophysics and in Saturn's ring in particular. While Larson was criticised for the many free parameters in his models, I think they are all introduced for good physical reasons, and his work is important in defining what sorts of star formation rates and friction coefficients are necessary. These are the food for other theorists to taste and think about, because we shall need an explanation of why the rates take values in Larson's range.

I agree with King that the problems of star formation are so little understood that 
the rest of the subject will be held back without more work theoretically and observationally on this problem.

It was very nice to see the advance of Technology making possible now what was almost impossible 20 years back. Thus Illingworth's velocity dispersions in globular clusters and Bertola's fine rotation curves for ellipticals and SOs are hints of what is to come. Lastly, it is always fun to mention the wilder speculations that can be the spice of life in science. Contopoulos in his review referred to Galgani's enthusiastic espousal of the theory of third and up to the $N$ th integral in a system of $N$ degrees of freedom at low energy. The $N$ adiabatic invariants there do give a basis for a sort of classical zero point energy. Can we still tell our students that classical mechanics has no explanations for the lowering of specific heats at low energy? If we still do so, we may well be telling less than the truth. It was also fun to see the close analogies between the classical theory and relativistic stellar dynamics represented by Ipser and Fackerell. It is sad that such a beautiful theory may not prove useful for real systems, but there is hope yet. The evolution of the core of an $N$ body system as described on the constant energy hypothesis does have the central potential behaving like $\psi_{0} \propto\left(t_{0}-t\right)^{-2 / 7}$. If we can find a large enough system of small enough stars there may yet be room in the world for relativistic clusters. 